\title{
A New Method To Measure The Noise Figure Of Multi-Channel Radar Receiving System
}

\author{
Weimei Li, Bo Liu \\ Xi'an Institute of Space Radio Technology, CAST, Xi 'an 710100, Shaanxi, China
}

Keywords: noise figure, NF measurement, multi-channel radar.

\begin{abstract}
Phased array radar or radar with array feed reflector antenna is made up of multiple channels, and the radar detection range is closely related to the noise figure (NF). Before the radar being put into operation, it's necessary to measure its NF to evaluate the system performance. Here, an NF measurement method of multi-channel radar receiving system is analyzed in detail with the theory of equivalent conversion of a multi-port network model into a two-port network model, combining with channel characteristics of array antenna with various amplitude weighting. To prove the correctness of the measurement method, a four-channel example is presented to show that the equivalent conversion method is feasible, and it also demonstrates that it is possible to precisely estimate NF of a multi-channel system through computation.
\end{abstract}

\section{Introduction}

Phased array radar or radar with array feed reflector antenna occupied an increasingly important position in the modern radar techniques. There usually exist complex feed network, delay lines and transmit/receive (TR) module in the radar, and such radar receiving system consists of one or multi-stage beam forming network together with the attenuator and the phase shifter etc. Noise can enter the radar receiver together with the echo signal through antenna or be generated by the radar receiver itself. For a microwave radar receiver, the external noise coming through the radar antenna is usually small, so the sensitivity of the receiver is mainly determined by its inherent noise, which is also referred to as internal noise. The noise level of radar receiver's internal noise is characterized by NF, and the radar detection range is closely related to NF shown in radar equation ${ }^{[1]}$.

In general, direct connection of an NF measuring instrument in the system under test can provide NF of a two-port receiver, but as for a radar receiving system with multiple channels, its NF can hardly be measured directly due to the multiple input ports. Furthermore, the NF of multi-channel radar with array feed reflector antenna is rarely discussed in previous work. For NF is the fundamental standard in designing a radar receiving system, it is necessary to compute the NF precisely. In the system trade-off study of a multi-channel radar, an accurate measurement of the overall noise figure is also necessary.
A figure of merit and $\mathrm{G} / \mathrm{T}$ for a multi-channel active array was derived by Lee ${ }^{[2]}$ in terms of noise performance, and the effects of the beam-forming network on the overall noise figure of the system was also studied. A general formulation for the computation of noise figure of active weighted array antennas is presented in [3]. An equivalent single-channel receiver model ${ }^{[4]}$ was used to analyze the beam sensitivity of an antenna array receiving system, in which efficiencies and noise temperature are parameters of the equivalent single-port antenna and equivalent amplifier. Two approaches ${ }^{[5,6]}$ were recently introduced for defining the efficiencies and system noise temperature of a receiving antenna array, one of which is based on the isotropic noise response of the array and the other on an equivalent system representation. However, none of the mentioned papers above involves the NF measurement method of a multi-channel radar receiving system. The relationship between radar system noise figure and channel noise figure is derived in [7], but the result is not applicable for multi-channel radar receiving system with amplitude weighting just like the radar with array feed reflector antenna. This paper presents a new NF measurement method which applies to every sort of the multi-channel radars. Here, as illustrated in Figure 1(a), a radar receiving system with array feed reflector antenna is showed to represent the multichannel receiving system, which consists of a number of branches with different weighting amplitudes and phases, and the phased array radar receiving system can be viewed as special case of Figure 1(a). To prove the correctness of the measurement method, the NF of the multi-channel radar receiving system is derived using the basic formulas proposed by [3].

\section{Noise characteristic analysis}

The array feed elements of Figure 1(a) (such as horns) usually have the same gain, however the input powers and phases of the echo from the reflector of the antenna into array feed element are different, the phases of each branch after the action of the phaser reached the combiner are also different unlike the phased array radar. All the phases of phased array radar in the receiving channels are controlled in a phase plane by the phase shifter, and the far field plane waves reached on the radiating elements are also in the same amplitude. We can set the output powers of each array element in Figure 1(a) are $\left(P_{1}, P_{2} \cdots P_{n}\right)$, and not all of them are the same. For the radar receiver excludes antenna in the common sense, the radar receiving system in Figure 1(a) can be simplified into Figure 
1(b), each channel can be represented by three components, a front-end attenuator $\left(\mathrm{L}_{1}\right)$, an LNA, and a back-end attenuator, here, we divided back-end attenuator into two parts: the fixed loss and the variable loss. The fixed loss includes ohmic loss of the attenuator, the phase shifter, and cables etc. The variable loss is the differential weightings amplitudes denoted by $L_{3 m}$. The LNA is characterized by a power gain $G_{A}$ and a noise figure $\mathrm{NF}_{\mathrm{A}}$. The front-end loss includes the attenuation in the circulator, limiter and cable losses etc. The ohmic loss of the combiner is denoted by $\mathrm{L}_{\mathrm{s}}$. Note that all the loss factors are numerically greater than 1 , and all the losses are truth values, not in decibel in the following formula derivation.

Based on the original definition of NF:

$$
N F=\frac{S_{\text {in }} / N_{\text {in }}}{S_{\text {out }} / N_{\text {out }}}=\frac{N_{\text {out }}}{N_{\text {in }}} \cdot \frac{S_{\text {in }}}{S_{\text {out }}}=\frac{N_{\text {out }}}{K T_{0} B} \cdot \frac{1}{G_{r}}
$$

where $K$ is Boltzmann constant, $T_{0}$ is the room temperature, B is the noise bandwidth of the device, $S_{\text {in }}$ and $S_{\text {out }}$ are the input and the output signal power of the device or a two-port receiver, respectively. $N_{i n}=K T_{0} B$ is the input noise, and $N_{\text {out }}$ is the noise power at the output end of the receiver, $G_{r}$ is power gain of the two-port receiver, if the receiver has multiple-port receiving network, it represents equivalent receiving gain of the receiver.

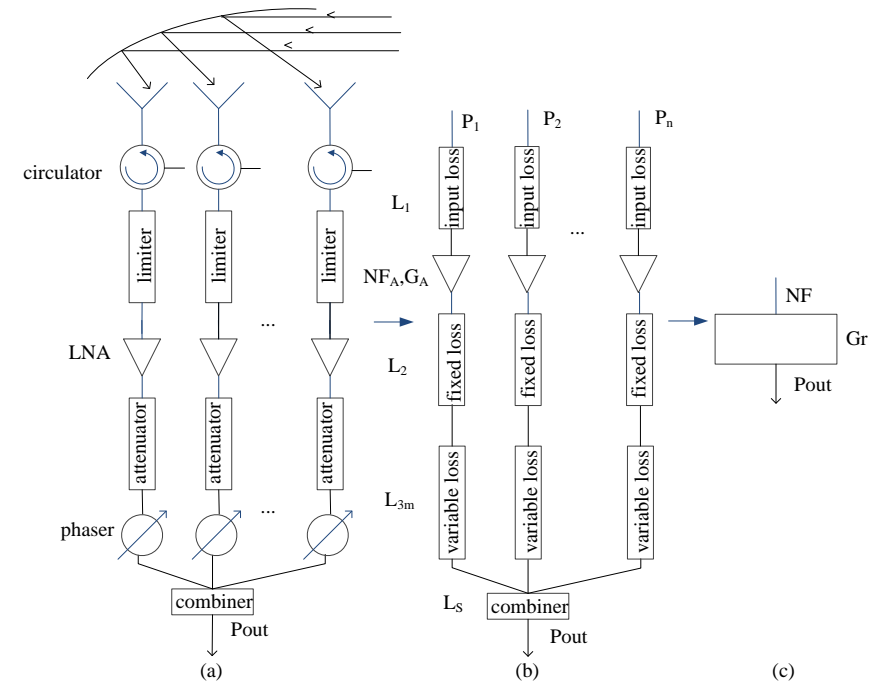

Figure 1: Simplification of the receiving system with array feed reflector antenna

The output noise power for each receiving channels before the combiner can be given as follows:

$$
N_{o m}=K T_{0} B \cdot N F_{m} \cdot G_{m}
$$

where $G_{m}$ is the power gain of the $m$ th receiving branch before the combiner, $m=1,2 \cdots n$, and $n$ is the number of the branches for the multiple-port radar receiving system. So $G_{m}=G_{A} /\left(L_{1} \cdot L_{2} \cdot L_{3 m}\right)$, from Figure $1(\mathrm{~b}), N F_{m}$ is the noise figure of the single $m$ th receiving branch before the combiner, $N F_{m}$ can be written as :

$$
N F_{m}=L_{1}+\frac{N F_{A}-1}{1 / L_{1}}+\frac{L_{2} L_{3 m}-1}{G_{A} / L_{1}}
$$

The additive output noise power introduced by the combiner can be given as below:

$$
N_{s}=\frac{K\left(L_{s}-1\right) T_{0} B}{L_{s}}
$$

The total output noise power at the output of the combining networks is as below:

$$
N_{o t}=\sum_{m=1}^{n} \frac{N_{o m}}{n L_{s}}+N_{s}
$$

The noise is incoherently summed(power sum), while the signals in the power combiner are combined in phase, thus are summed coherently.

The total input signal power $S_{i n}$ in Figure 1(b) is $S_{i n}=\sum_{m=1}^{n} P_{m}$, and output signal power for $m$ th receiving channel reaches the output of the combiner can be given as follows:

$$
S_{\text {om }}=P_{m} G_{m} /\left(n L_{s}\right)
$$

Assuming that the final phase of each channel at the input end of the combiner is $\theta_{m}$ after the phases shifting, the total output signal power at the output of the combining networks is given as follows:

$$
S_{\mathrm{o}}=\left|\sum_{m=1}^{n} \sqrt{S_{o m}} e^{j \theta_{m}}\right|^{2}
$$

Thus we can get the equivalent receiving gain:

$$
G_{r}=\frac{S_{o}}{S_{i n}}=\frac{G_{A}}{n L_{1} L_{2} L_{s}} \frac{\left|\sum_{m=1}^{n}\left(\sqrt{\frac{P_{m}}{L_{3 m}}}\right) e^{j \theta_{m}}\right|^{2}}{\sum_{m=1}^{n} P_{m}}
$$

So far, the receiving system in Figure 1(a) can be simplified into Figure 1(c), with the equivalent gain $G_{r}$ and noise figure of $N F$. Based on the definition of noise figure in Eq. (1), the noise figure of the multi-channel radar receiving system is expressed as follows:

$$
\begin{aligned}
& N F=\frac{N_{o t}}{N_{i n}} \cdot \frac{1}{G_{r}}=\frac{\sum_{m=1}^{n}\left(K T_{0} B \cdot N F_{m} \cdot G_{m} \cdot \frac{1}{n L_{s}}\right)+N_{s}}{K T_{0} B} \cdot \frac{1}{G_{r}} \\
& =\left(L_{1} N F_{A} \sum_{m=1}^{n} \frac{1}{L_{3 m}}-\frac{L_{1}}{G_{A}} \sum_{m=1}^{n} \frac{1}{L_{3 m}}+\frac{n L_{1} L_{2} L_{s}}{G_{A}}\right) \cdot \frac{\sum_{m=1}^{n} P_{m}}{\left|\sum_{m=1}^{n}\left(\sqrt{\frac{P_{m}}{L_{3 m}}}\right) e^{j \theta_{m}}\right|^{2}}
\end{aligned}
$$

Note that, the power $P_{m}$ in Eq. (9) does influence the NF, and it represents the signal reflected from the antenna reflector. The input signal of the receiving system is the far-field plane wave from the scatterers. Because the reflector is part of the 
receiving system, so the NF result is still not related to the input power. In the special case, this formula also applies to the phased array radar receiving system. For a phased array radar, the input power $P_{1}=P_{2}=\cdots=P_{n}$, and the phase at the input end of the combiner is $\theta_{1}=\theta_{2}=\cdots=\theta_{n}$.

\section{Noise figure measurement}

Noise figure usually represents the performance of a two-port receiver, and its measurement is also based on a two-port network, as for the radar receiving system as show in Figure 1(a), the following will discuss its measurement method. Based on the analysis in section 2, a multi-port network shown in Figure 1(a) can be converted into a two-port network shown in Figure 1(c), an arbitrary power divider with ohmic loss denoted as Lg can be added in the input end of the two-port network in Figure 1(c), and the receiving system in Figure 1(b) is turn into be what shown in Figure 2(a), all the component are the same as what in Figure 1(b) except for the power divider.

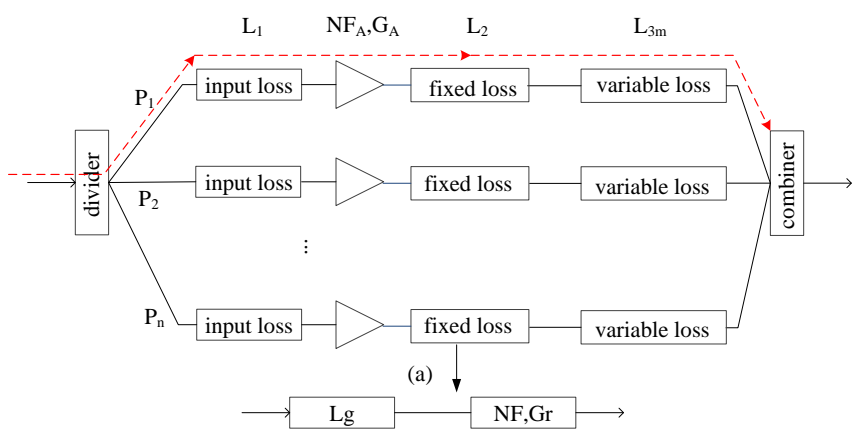

(b)

Figure 2: Equivalent measurement diagram of the receiving system

Based on the previous analysis, we can assume that the system in Figure 2(a) with $\mathrm{Lg}=0$ is just the system in Figure 1(b), that is NF of Figure 2(b) with $\mathrm{Lg}=0$ is the same as that of Figure 1(c) .To confirm this hypothesis, we compute the noise figure in Figure 2(a).

In Figure 2(a), set the input signal power is $S_{i n}{ }^{\prime}$, and the signal power at the $m$ th input port of each channel marked in the dashed line is $P_{m}$, for the signals after the arbitrary power divider are different. The relationship of them is $S_{i n}{ }^{\prime}=\sum_{m=1}^{n} P_{m}$. The power gain of the $m$ th receiving branch, before the combiner is:

$$
G_{m}{ }^{\prime}=G_{A} /\left(L_{g} \cdot L_{1} \cdot L_{2} \cdot L_{3 m}\right)
$$

Output signal power for $m$ th receiving channels reaches the output of the combiner is as follows:

$$
S_{\text {om }}{ }^{\prime}=P_{m} G_{m}{ }^{\prime} /\left(n L_{s}\right)
$$

The total output signal power at the output of the combining networks is given as follows:

$$
S_{\mathrm{o}}{ }^{\prime}=\left|\sum_{m=1}^{n} \sqrt{S_{o m}{ }^{\prime}} e^{j \theta_{m}{ }^{\prime}}\right|^{2}
$$

where $\theta_{m}{ }^{\prime}$ is the final modulated phase after phase shifting at the input end of combiner for each channel in Figure 2(a), and the equivalent receiving gain can be got:

$$
G_{r}{ }^{\prime}=\frac{S_{o}{ }^{\prime}}{S_{i n}{ }^{\prime}}=\frac{G_{A}}{n L_{g} L_{1} L_{2} L_{s}} \frac{\left|\sum_{m=1}^{n}\left(\sqrt{\frac{P_{m}}{L_{3 m}}}\right) e^{j \theta_{m}{ }^{\prime}}\right|^{2}}{\sum_{m=1}^{n} P_{m}}
$$

Though the output signals after the power divider in Figure 2(a) are in phase, and the phases at the input of each channel in Figure 1(b) are different, nevertheless, the phases being considered in the formulas are both the final results after phases shifting at each input end of combiner in the both figures, thus $\theta_{m}$ ' can be equal to $\theta_{m}$.

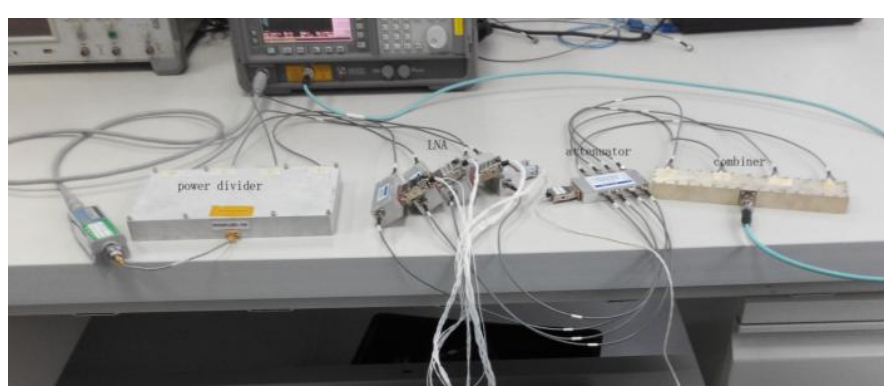

Figure 3: Measurement of a four-branch equivalent experiment system

The total input noise power in Figure 2(a) is $N_{i n}{ }^{\prime}=K T_{0} B$, and the noise power at the input end of channel marked in red dashed line is $N_{1}=N_{2}=\cdots=N_{m}=K T_{0} B$, whether the power divider is arbitrary or equal, because the input noise power can be assumed as thermal noise, so they are same for all branches.

The noise figure of the single $m$ th receiving branch before the combiner in Figure 2(a) can be written as :

$$
N F_{m}{ }^{\prime}=L_{g}+\frac{L_{1}-1}{1 / L_{g}}+\frac{N F_{A}-1}{1 / L_{1} L_{g}}+\frac{L_{2} L_{3 m}-1}{G_{A} / L_{1} L_{g}}
$$

The output noise power for each receiving channels before the combiner can be given as follows:

$$
N_{o m}{ }^{\prime}=K T_{0} B \cdot N F_{m}{ }^{\prime} \cdot G_{m}{ }^{\prime}
$$

The additive output noise power introduced by the combiner can be given as below:

$$
N_{s}=\frac{K\left(L_{s}-1\right) T_{0} B}{L_{s}}
$$

The total output noise power at the output of the combining networks in Figure 2(a) is as below: 


$$
N_{o t}{ }^{\prime}=\sum_{m=1}^{n} \frac{N_{o m}{ }^{\prime}}{n L_{s}}+N_{s}
$$

Substituting the Eq. (13) and Eq. (17) in to Eq. (1), we will get the noise figure of the system as shown in Figure 2(a).

$$
\begin{aligned}
& N F^{\prime}=\frac{N_{o t}{ }^{\prime}}{N_{i n}{ }^{\prime}} \cdot \frac{1}{G_{r}{ }^{\prime}}= \\
& \left(L_{1} N F_{A} \sum_{m=1}^{n} \frac{1}{L_{3 m}}-\frac{L_{1}}{G_{A}} \sum_{m=1}^{n} \frac{1}{L_{3 m}}+\frac{n L_{1} L_{2} L_{s}}{G_{A}}\right) \cdot \frac{L_{g} \cdot \sum_{m=1}^{n} P_{m}}{\left|\sum_{m=1}^{n}\left(\sqrt{\frac{P_{m}}{L_{3 m}}}\right) e^{j \theta_{m}}\right|^{2}}
\end{aligned}
$$

Comparing Eq. (9) with the Eq. (18), it is clearly that the NF results of measurement system in Figure 2(a) is $\mathrm{Lg}$ (if in decibel) larger than the actual radar system for the introduction of the power divider. To verify the correctness of our research result, a four-branch measurement model as Figure 2(a) is built in Figure 3. The ohmic loss of the power divider is $L_{g}=1 \mathrm{~dB}$, the $\mathrm{S}$ parameters of the divider are $S_{21}=S_{41}=-6 \mathrm{~dB}, S_{31}=-4.5 \mathrm{~dB}$ and $S_{51}=-8.5 \mathrm{~dB}, L_{1}=0.9 \mathrm{~dB}$, $L_{2}=8.3 \mathrm{~dB}, L \mathrm{~s}=0.4 \mathrm{~dB}, G_{A}=30 \mathrm{~dB}, N F_{A}=1.4 \mathrm{~dB}, L_{3 \mathrm{~m}}=[31.5,31.5$, 10, 4.5]dB. In computation NF in Figure 3 by Eq. (18), the ohmic losses of attenuator and cables are included in $L_{2}$, the four weightings amplitudes from the attenuator are $L_{3 \mathrm{~m}}$.

\begin{tabular}{|c|c|c|}
\hline & $N F^{\prime}$ & $G_{r^{\prime}}$ \\
\hline Computation & 7.792 & 5.676 \\
\hline Measurement & 7.67 & 5.71 \\
\hline
\end{tabular}

Table 1: Computation and measurement result $(\mathrm{dB})$.

It can be seen from Table 1 that computation and measurement results from the equivalent measurement system are basically in agreement, the little error is due to precision of instrument and environmental influences of experiment. The measurement data plus or minus the ohmic loss of the power divider resulting the gain and NF of the multi-channel radar receiving system based on previous analysis. So the NF and gain of the four-branch radar receiving network as shown in Figure 1(a) are to be $6.67 \mathrm{~dB}$ and $6.71 \mathrm{~dB}$, respectively.

\section{Conclusion}

The theory of equivalent conversion of a multi-port network model into a two-port network model, is used to measure NF and gain of multi-channel radar receiving system, and the NF and gain of the equivalent system are also analyzed in detail to demonstrate the correctness of the measurement method together with the measurement of a four-way network. We can directly measure NF and gain of a multi-port network with the help of a power divider, and the measurement data plus or minus the ohmic loss of the power divider resulting the gain and NF of the multi-channel radar receiving system.

\section{Acknowledgements}

This work was supported by Xi'an Institute of Space Radio Technology.

\section{References}

[1] Skolnik, Merrill I., "Introduction to Radar Systems," 3rd Edition, McGraw Hill Inc., (2001).

[2] Lee J J, "G/T and noise figure of active array antennas," IEEE Trans. Antennas Propagat., 41, pp. 241-244, (1993).

[3] Gatti R V, Dionigi M, and Sorrentino R, "Computation of Gain, Noise Figure, and Third-Order Intercept of Active Array Antennas," IEEE Trans. Antennas Propagat., 52, pp. 3139-3142, (2004).

[4] Ivashina M V, Maaskant R, and Woestenburg B, "Equivalent system representation to model the beam sensitivity of receiving antenna arrays," IEEE Antennas Wireless Propag. Lett., 7, pp. 733-737, (2008).

[5] Warnick K F and Jeffs B D, "Efficiencies and system temperature for a beam forming array," IEEE Antennas Wireless Propag. Lett., 7, pp. 565-568, (2008).

[6] Warnick K F, Ivashina M V, Maaskant R, and Woestenburg B, "Unified definitions of efficiencies and system noise temperature for receiving antenna arrays," IEEE Trans. Antennas Propagat., 58, pp. 2121-2125, (2010).

[7] Lai H, Zhou J and Li B, "Noise figure measurement of multichannel phased array receiver module," 2011 IEEE 4th International Symposium on Microwave, Antenna, Propagation, and EMC Technologies for Wireless Communications (MAPE), pp. 232-235, (2011). 